\title{
Fixed Eigenvector Analysis of Thermographic NDE Data
}

\author{
K. Elliott Cramer ${ }^{*}$ and William P. Winfree \\ NASA Langley Research Center \\ Hampton, VA 23681
}

\begin{abstract}
Principal Component Analysis (PCA) has been shown effective for reducing thermographic NDE data. This paper will discuss an alternative method of analysis that has been developed where a predetermined set of eigenvectors is used to process the thermal data from both reinforced carbon-carbon (RCC) and graphite-epoxy honeycomb materials. These eigenvectors can be generated either from an analytic model of the thermal response of the material system under examination, or from a large set of experimental data. This paper provides the details of the analytic model, an overview of the PCA process, as well as a quantitative signal-to-noise comparison of the results of performing both conventional PCA and fixed eigenvector analysis on thermographic data from two specimens, one Reinforced Carbon-Carbon with flat bottom holes and the second a sandwich construction with graphite-epoxy face sheets and aluminum honeycomb core.
\end{abstract}

Keywords: thermography, reinforced carbon-carbon, signal-to-noise, principal component analysis

\section{INTRODUCTION}

Principal component analysis (PCA) has been used extensively as a data reduction technique. Originated by Peason ${ }^{1}$ in 1901 and later developed more fully by Hotelling ${ }^{2}$ it has been used extensively to reduce the dimensionality of a data set consisting of a large number of interrelated variables, while retaining, as much as possible, the variation present in the data set. For example PCA is used routinely in constructing socio-economic status indices ${ }^{3}$, for analysis of spectral imaging data in astrophysics ${ }^{4}$ and for facial recognition ${ }^{5}$. Additionally, application of PCA to thermal NDE has also been extensively studied. Typical application of PCA to the reduction of transient thermographic data consists of calculating the principal components of the temporal data through singular value decomposition (SVD) of the experimental data itself. For example, Rajic ${ }^{6,7}$ and Valluzzi ${ }^{8}$ both use PCA as a contrast enhancement technique for defect detection. Genest ${ }^{9}$ and Vavilov ${ }^{10}$ provide comparisons between PCA and various other data reduction techniques for defect sizing. Zalameda ${ }^{11}$ discusses PCA's use for temporal compression of the thermal data. PCA was used to analyze thermal "flying-spot" data by Hermosilla-Lara ${ }^{12}$ for detection of open cracks in metallic specimens. Finally, Marinetti ${ }^{13}$ suggests the use of a experimentally derived training set to calculate the principal components. This paper reports on the use of a semi-analytical, onedimensional model to develop the principal components. The application of this approach to the inspection of two material types will be examined as test cases: (1) Reinforced carbon-carbon (RCC) material, which can realistically be model as a one-dimensional response and (2) honeycomb sandwich materials with graphite-epoxy face sheets and aluminum core which typically requires a two-dimensional model to accurately represent the thermal response. The

*k.elliott.cramer@nasa.gov; phone 757-864-7945; fax 757-864-4914 
latter test case will examine whether a simplified one-dimensional model can be used to generate an acceptable set of fixed principal components.

\section{EXPERIMENTAL EQUIPMENT}

The flash thermography system performing these measurements was a commercially available system. The system features an infrared (IR) imager with a 320 x 256 sensor array of Indium-Antimonide elements and two 4800-Joule xenon photographic flash tubes mounted in a hood to contain and focus the flash. The hood has dimensions of 36.8 $\mathrm{cm}$ wide by $26.7 \mathrm{~cm}$ deep by $40.6 \mathrm{~cm}$ tall and is configured such that the IR camera views the inspection surface directly. The flash produces a flash energy density of 7.15 joules per square centimeter at the mouth of the hood ${ }^{14}$. The inspection hood is connected to a computer base station that also houses power sources for various components. Thermographic inspection was accomplished by placing the hood on the section of material to be inspected. The bottom of the hood completely surrounds or sits on top of the specimen, depending on the dimension of the specimen as compared to the size of the hood. The flash lamps are triggered either by operator controls on the hood or by the computer. Thermographic images of the specimen are captured by the infrared camera for a predetermined amount of time and stored in the computer for further analysis. The camera's noise equivalent temperature difference $(\mathrm{NE} \Delta \mathrm{T})$, cited by the manufacturer, is $0.025^{\circ} \mathrm{C}$ in the 3 to 5 micrometer wavelength range. External optics, consisting of a wide-angle lens, using germanium optical elements, was used to increase the system field-ofview by a factor of approximately two. The expanded field-of-view of this lens is $41^{\circ}$ horizontally and $31^{\circ}$ vertically.

\section{DATA ANALYSIS}

The algorithm used to perform the PCA is based on the decomposition of the thermal data into its principal components or eigenvectors using SVD. PCA is performed by first reformatting the three-dimensional thermal data into a two-dimensional array where the columns contain the spatial information and the rows contain the temporal information such that $T(x, y, t)$ becomes $A(n, m)$ where $n=N_{x} * N_{y}$ and $m=N_{t}$. The matrix $A$ is then adjusted by subtracting the mean along the time dimension, and decomposed to yield the eigenvalues and eigenvectors:

$$
A=U \Gamma V^{T}
$$

where $U$ and $V$ are orthogonal matrices who's columns form the eigenvectors of $\mathrm{AA}^{\mathrm{T}}$ and $\mathrm{A}^{\mathrm{T}} \mathrm{A}$ respectively and $\Gamma$ contains the singular values (the nonnegative square roots of the eigenvalues) of $A^{\mathrm{T}} \mathrm{A}$. Since the columns of $\mathrm{U}$ corresponding to nonzero singular values form an orthogonal basis for the range space of $\mathrm{A}$, the entire thermal data set can be described by this basis. Because thermal NDE signals are well behaved and slowly varying in time, the predominant temporal variations of the entire data set are usually contained in the first and second eigenvector. The PCA images are formed by calculating the dot product of the measured temperature response, pixel by pixel, with the eigenvectors of interest (usually the two associated with the largest eigenvalues). Defects in the material under investigation change the local temporal variation of the data and thus appear as either light or dark regions in the PCA images.

While this process is quite effective in reducing thermal data, the SVD can be computationally intense especially with large three-dimensional arrays of thermal data typically produced during an inspection. In order to reduce the computation time and enhance the performance, the PCA reduction was performed using a fixed set of previously generated eigenvectors. Two different approaches to generating the fixed eigenvectors were investigated. First, an array of thermal responses of the material was calculated from a one dimensional multilayer analytic model. A set of eigenvectors were then numerically generated from this array of responses. A second set of eigenvectors were numerically generated from the thermal responses measured on an assortment of specimens with thicknesses approximately equal to the structure of interest. 


\section{ONE DIMENSIONAL MODEL}

To simulate the front surface temperature response of the RCC material to a flash heat input, a one dimensional, multi-layer model was developed to solve the heat equation. A classic Laplace transform approach was taken to solve the heat equation. The heat equation for a one dimensional slab of finite thickness is:

$$
\frac{\partial^{2} T}{\partial x^{2}}=\frac{1}{\alpha} \frac{\partial T}{\partial t}
$$

where $T$ is temperature, $t$ is time, $x$ is the dimension normal to the surface of the slab with $x=0$ being the slab face of interest and $\alpha$ is the thermal diffusivity. The heat flux, $\Phi$, is assumed for this model to be a Dirac heat pulse of total energy $Q$ and is applied at $x=0$ with an insulating back surface $(x=d)$ where $d$ is the thickness of the layer. In the time domain this leads to the following boundary conditions:

$$
\begin{gathered}
\Phi_{0}=Q \delta(t) \text { at } \mathrm{x}=0 \text { and } \\
\Phi_{d}=0 \text { at } \mathrm{x}=\mathrm{d} .
\end{gathered}
$$

Applying a Laplace transform to the heat equation and assuming the initial temperature is zero yields:

$$
\frac{d^{2} \theta(x, P)}{d x^{2}}=\frac{P}{\alpha} \theta(x, P),
$$

where $P$ is the Laplace parameter and $\theta$ is given by:

$$
\theta(x, P)=\int_{0}^{\infty} e^{-P t} T(x, t) d t .
$$

The boundary conditions can also be transformed into the Laplace domain as:

$$
\begin{gathered}
\phi_{0}=Q \text { at } \mathrm{x}=0 \text { and } \\
\phi_{d}=0 \text { at } \mathrm{x}=\mathrm{d} .
\end{gathered}
$$

It is typical to write the four quantities of interest $\theta_{0}, \phi_{0}, \theta_{\mathrm{d}}$, and $\phi_{\mathrm{d}}$, the front face Laplace temperature and flux and the Laplace temperature and flux at $\mathrm{x}=\mathrm{d}$ respectively, in matrix form as:

$$
\left[\begin{array}{l}
\theta_{d} \\
\phi_{d}
\end{array}\right]=\left[\begin{array}{ll}
A & B \\
C & D
\end{array}\right]\left[\begin{array}{l}
\theta_{0} \\
\phi_{0}
\end{array}\right],
$$

where, 


$$
\left[\begin{array}{ll}
A & B \\
C & D
\end{array}\right]=\left[\begin{array}{cc}
\cosh (q d) & -\frac{1}{q K} \sinh (q d) \\
-K q \sinh (q d) & \cosh (q d)
\end{array}\right]
$$

where

$$
q=\sqrt{P / \alpha}
$$

and $\mathrm{K}$ is the thermal conductivity of the layer.

A material with a thickness of $\mathrm{d}$ consisting of $\mathrm{n}$ layers of thickness $l_{\mathrm{i}}$ with thermal conductivities and diffusivities of $\mathrm{K}_{\mathrm{i}}$ and $\alpha_{\mathrm{i}}$ can be expressed as a product of matrices, in the Laplace domain, as follows:

$$
\begin{aligned}
{\left[\begin{array}{l}
\theta_{d} \\
\phi_{d}
\end{array}\right]=} & {\left[\begin{array}{cc}
A_{n} & B_{n} \\
C_{n} & D_{n}
\end{array}\right]\left[\begin{array}{cc}
1 & -R_{n-1} \\
0 & 1
\end{array}\right] \ldots } \\
& {\left[\begin{array}{cc}
1 & -R_{1} \\
0 & 1
\end{array}\right]\left[\begin{array}{cc}
A_{1} & B_{1} \\
C_{1} & D_{1}
\end{array}\right]\left[\begin{array}{l}
\theta_{o} \\
\phi_{0}
\end{array}\right] }
\end{aligned}
$$

where

$$
\begin{gathered}
{\left[\begin{array}{ll}
A_{i} & B_{i} \\
C_{i} & D_{i}
\end{array}\right]=\left[\begin{array}{cc}
\cosh \left(q_{i} l_{i}\right) & -\frac{1}{q_{i} K_{i}} \sinh \left(q_{i} l_{i}\right) \\
-K_{i} q_{i} \sinh \left(q_{i} l_{i}\right) & \cosh \left(q_{i} l_{i}\right)
\end{array}\right]} \\
q_{i}=\sqrt{P / \alpha_{i}}
\end{gathered}
$$

and $R_{n}$ is a contact resistance between adjacent layer to simulate a defect (for example the thermal conductivity and thickness of an air gap could be used to calculate R) that would reduce the rate of heat flow. Once the matrix has been established, then numeric methods are used to perform the inverse Laplace transform back into the time domain using the de Hoog algorithm ${ }^{15}$ and Matlab® code by Hollenbeck ${ }^{16}$.

\section{CASE STUDIES}

\subsection{Reinforced Carbon Carbon}

The RCC material of interest is a layered structure consisting of Silicon Carbide ( $\mathrm{SiC}$ ) on the front and back surfaces and reinforced carbon-carbon in the middle. Additionally, most specimens have other coatings of various types applied to the outer (front) surface. Because of the nonuniformity of the SiC layer as well as the complex nature of this material, a four layer model was chosen to approximate the thermal response. Layer one was SiC, layer two was an amalgam of the material properties of $\mathrm{SiC}$ and carbon-carbon providing a transition region, layer three was carbon-carbon and layer four was $\mathrm{SiC}$.

For all cases a unitary Dirac input heat flux $(\mathrm{Q})$ was assumed to be a good approximation for the input flux, since the pulse duration is short relative to the thermal response time of the specimen. 
Figure 1a shows the front surface temperature as predicted by the analytic model. The output of the model and the experimental results were both normalized for comparison. Figure $1 \mathrm{~b}$ shows the difference between the model and the experimental data as a function of time, indicating that agreement of better than $99 \%$ is achieved. To provide a statistically rich data set for the calculation of the eigenvectors, a family of 10,000 characteristic curves was produced using the one-dimensional model. The curves represent a series of thickness variations of $\pm 10 \%$ for each of the first three layers of the model, but maintained the overall thickness of the specimen.

The primary specimen used for validation was a $15 \mathrm{~cm}$ square RCC specimen with 18 flat-bottom holes at five varying diameters and depths. Figure 2 illustrates the size, depth, and location of the holes as observed from the back side of the sample. The depths of the defects ranged from material loss only in the silicon carbide layer (row $\mathrm{E}$ in Figure 2) to holes $92 \%$ through the full thickness of the sample (row A). Flash thermography was performed on this specimen using the system previously described. Data was collected at a frame rate of 60 frames per second for 14 seconds after the flash heating. Processed images were then calculated using the three approaches, over two different time windows ( 0.16 to 3.5 seconds and 1 to 11.5 seconds after heating $)^{14,17-18}$. Figure 3 shows the typical results obtained with processing of the thermal data using three different methods, eigenvectors calculated on the acquired data (PCA), model-based fixed eigenvectors and experimental-based fixed eigenvectors respectively.

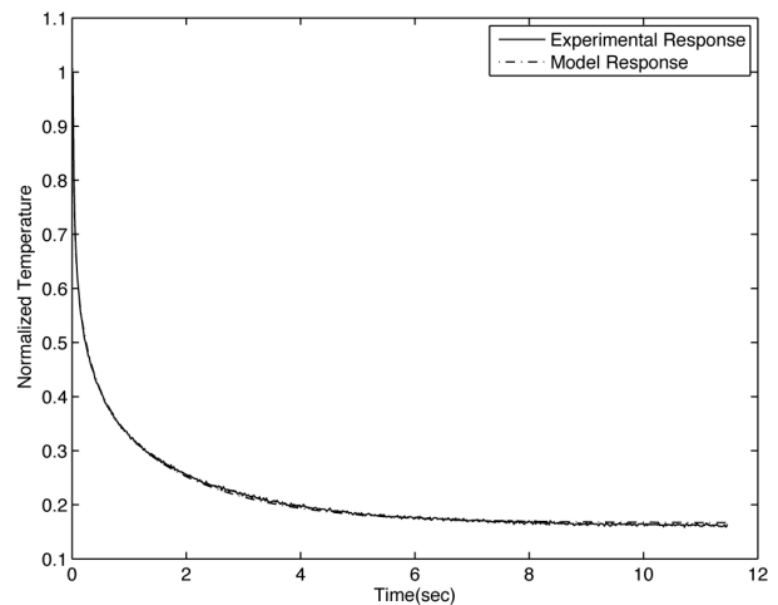

(a)

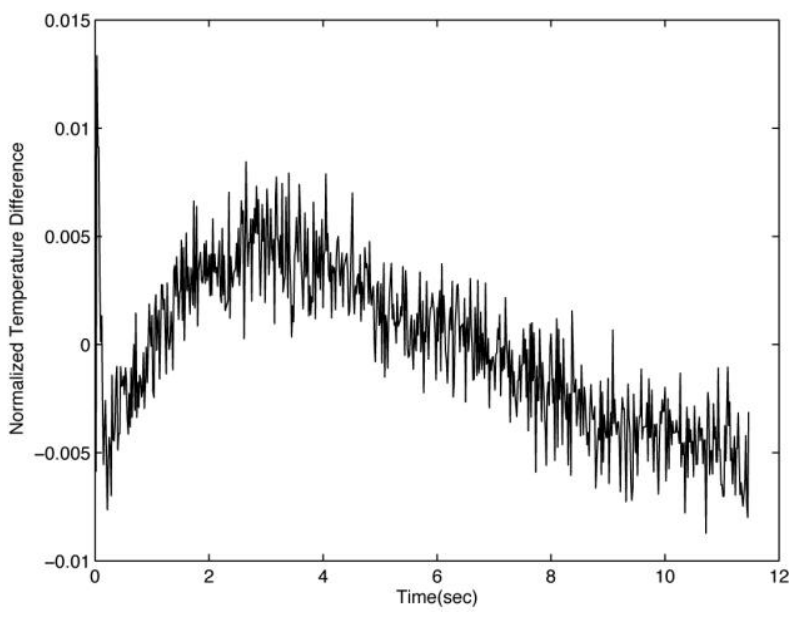

(b)

Figure 1. (a) Results of the four layer 1-D analytic model results with the experimental response of RCC material, and (b) the difference between experimental results and analytic model of RCC material showing better than $99 \%$ agreement.

To quantitatively compare the results of the three approaches the signal-to-noise ratio (SNR) of the difference between the defect signal and background signal was calculated for each of the 18 defects in both the early and late time windows. A defect signal was determined by averaging the signal from a $3 \times 3$ pixel area in the center of each flat-bottom hole. A background signal was determined by averaging the signal from a circle of $2.54 \mathrm{~cm}$ in diameter around the same defect. The pattern used for the SNR calculation is shown graphically in Figure 4. The SNR was then determined from the following equation:

$$
S N R=\frac{S^{\text {defect }}-S^{\text {background }}}{\sigma^{\text {background }}}
$$

where $S$ represents the average of the pixel values and $\sigma$ represents the standard deviation of the pixel values. 
In general it can be seen from Table 1, which compares the three techniques, that greater signal-to-noise ratios are obtainable using the fixed eigenvector PCA approach. Both the model-based and experimental-based fixed eigenvector approaches produce comparable increases in the SNR over the conventional technique. The average increases in the SNR for the model-based approach was 248\% and for the experimentally based approach $360 \%$. This is especially true for the deeper defects such as E2, E3, D3, E4 and D5, where the fixed eigenvector approach increased the SNR to above 1.0. The SNRs shown in Table I are the largest values obtained by either technique, for either time window at each defect location.

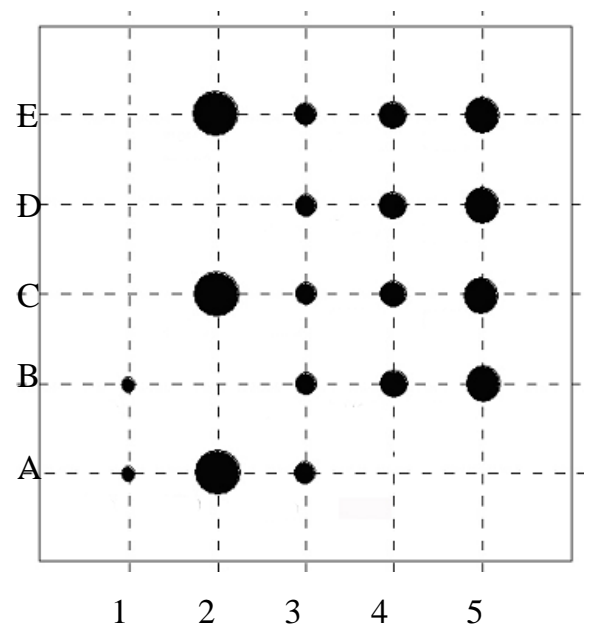

(a)

\begin{tabular}{|c|c|c|c|c|c|}
\hline & 1 & 2 & 3 & 4 & 5 \\
\hline A & $\begin{array}{c}0.318 \mathrm{~cm} \\
92 \%\end{array}$ & $\begin{array}{c}1.27 \mathrm{~cm} \\
52 \%\end{array}$ & $\begin{array}{c}0.635 \mathrm{~cm} \\
88 \%\end{array}$ & & \\
\hline B & $\begin{array}{c}0.318 \mathrm{~cm} \\
76 \%\end{array}$ & & $\begin{array}{c}0.635 \mathrm{~cm} \\
72 \%\end{array}$ & $\begin{array}{c}0.476 \mathrm{~cm} \\
68 \%\end{array}$ & $\begin{array}{c}0.953 \mathrm{~cm} \\
72 \%\end{array}$ \\
\hline $\mathrm{C}$ & & $\begin{array}{c}1.27 \mathrm{~cm} \\
24 \%\end{array}$ & $\begin{array}{c}0.635 \mathrm{~cm} \\
48 \%\end{array}$ & $\begin{array}{c}0.476 \mathrm{~cm} \\
48 \%\end{array}$ & $\begin{array}{c}0.953 \mathrm{~cm} \\
48 \%\end{array}$ \\
\hline $\mathrm{D}$ & & & $\begin{array}{c}0.635 \mathrm{~cm} \\
24 \%\end{array}$ & $\begin{array}{c}0.476 \mathrm{~cm} \\
24 \%\end{array}$ & $\begin{array}{c}0.953 \mathrm{~cm} \\
24 \%\end{array}$ \\
\hline $\mathrm{E}$ & & $\begin{array}{c}1.27 \mathrm{~cm} \\
12 \%\end{array}$ & $\begin{array}{c}0.635 \mathrm{~cm} \\
12 \%\end{array}$ & $\begin{array}{c}0.476 \mathrm{~cm} \\
12 \%\end{array}$ & $\begin{array}{c}0.953 \mathrm{~cm} \\
8 \%\end{array}$ \\
\hline
\end{tabular}

(b)

Figure 2. (a) Drawing of RCC flat-bottom hole specimen and (b) table of flaw diameters and flaw depths as a fraction of the total specimen thickness.

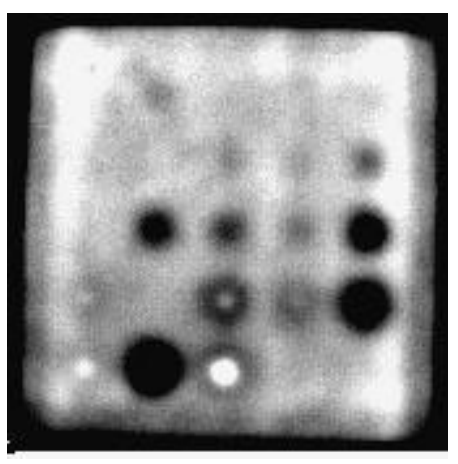

(a)

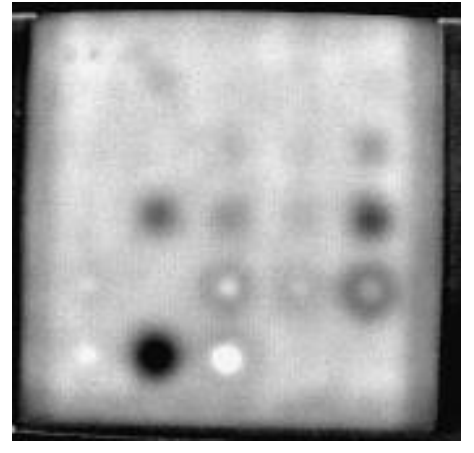

(b)

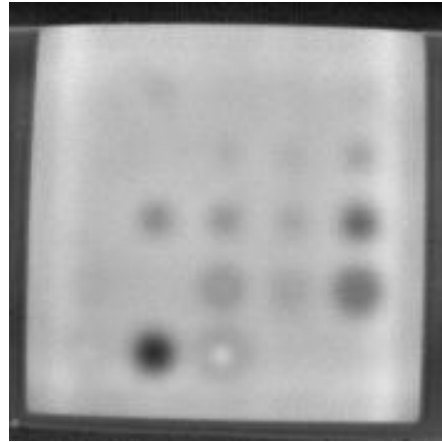

(c)

Figure 3. Typical processed results of the flat-bottom-hole specimen. (a) Eigenvectors calculated from acquired data, (b) model-based fixed eigenvectors and (c) experimental-based fixed eigenvectors. These results were achieved using the 0.16 to 3.5 second time window. 


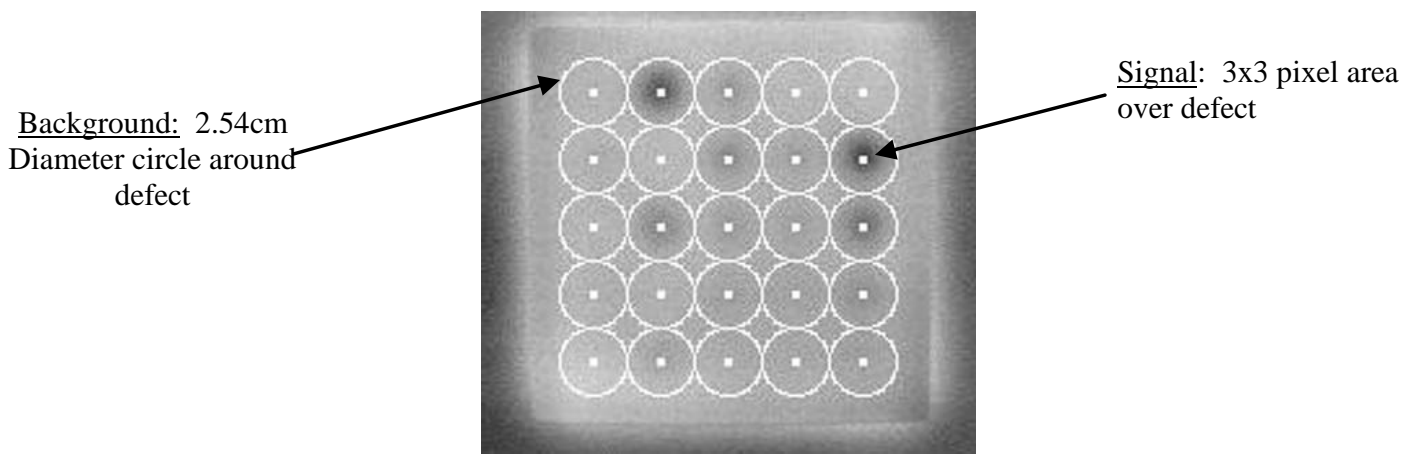

Figure 4. Graphical representation of the regions used for the SNR calculation for the defects in the flat-bottom-hole specimen.

Table 1. Maximum signal-to-noise ratios for the 18 defects of the RCC calibration specimen calculated from the model-based and experimental-based fixed eigenvector methods as well as conventional PCA.

\begin{tabular}{|c|c|c|c|}
\hline Defect Number & $\begin{array}{c}\text { Model-Based PCA } \\
\text { Max SNR }\end{array}$ & $\begin{array}{c}\text { Exp-Based PCA } \\
\text { Max SNR }\end{array}$ & $\begin{array}{c}\text { Conventional PCA } \\
\text { Max SNR }\end{array}$ \\
\hline B1 & 6.3867 & 7.7112 & 2.4165 \\
\hline A1 & 2.5937 & 1.4812 & 10.3391 \\
\hline E2 & 1.9813 & 2.76722 & 0.2155 \\
\hline C2 & 6.7688 & 1.94191 & 4.7612 \\
\hline A2 & 11.134 & 52.4278 & 13.8615 \\
\hline E3 & 1.0056 & 1.24770 & 0.8833 \\
\hline D3 & 2.4172 & 4.3308 & 0.5044 \\
\hline C3 & 7.9868 & 2.13199 & 2.5116 \\
\hline B3 & 11.0751 & 71.1625 & 20.032 \\
\hline A3 & 11.9819 & 24.6237 & 23.7523 \\
\hline E4 & 1.1182 & 0.825309 & 0.3772 \\
\hline D4 & 4.767 & 3.25975 & 1.2643 \\
\hline C4 & 7.9 & 6.41337 & 3.0336 \\
\hline B4 & 7.5803 & 10.2903 & 5.5433 \\
\hline E5 & 1.5343 & 1.07745 & 1.0771 \\
\hline D5 & 3.3425 & 3.10497 & 0.4911 \\
\hline C5 & 8.2382 & 12.9393 & 8.5228 \\
\hline B5 & 6.1298 & 256.733 & 9.2124 \\
\hline
\end{tabular}




\subsection{Honeycomb Sandwich}

The sandwich material consists of a $0.1 \mathrm{~cm}$ thick graphite-epoxy (GrEp) skin over a $2.54 \mathrm{~cm}$ thick aluminum honeycomb core. A four layer model (two layers of GrEp, one layer each of air and aluminum) was used to simulate the thermal response of the sandwich material, with a variable thickness air layer between the skin and the core used to model skin-to-core disbonding. Additionally, the contact resistance $\left(R_{n}\right.$ in equation 12) and the thickness of the two layers of GrEp skin were varied to model disbonding within the skin. Since the sandwich material geometry is more complex than can be captured in a one dimensional model, the model was initially used to bound the thermal response. Figure 5 shows the both the average experimental thermal response over a large area of sandwich material and the computational results for single layer GrEp skin and for GrEp over solid aluminum. As anticipated the experimental results lie between the two modeled conditions. In order to form a statistically rich set of data for the fixed eigenvectors in this case, the four layer model was run 10,000 times to produce a family of characteristic curves that model disbonding in the GrEp skin and at the skin-aluminum interface.

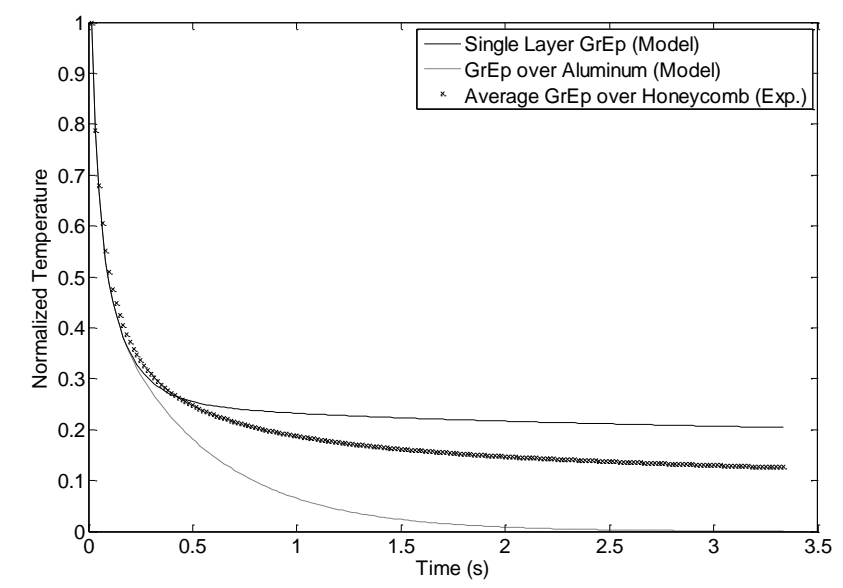

Figure 5. Comparison of experimental results from a honeycomb sandwich composite specimen with two one-dimensional model results (GrEp skin only and GrEp skin over solid aluminum).

The honeycomb specimen type examined with this technique is shown in Figure 6 and an exploded view is shown in Figure 7. The specimen is 7.6- $\mathrm{cm}$ wide and 61.2-cm long. Two separate sandwich sections are joined together by 12.7-cm long tapered composite splices located on opposite sides of the specimen in the center. Two solid graphite/epoxy laminate blocks are bonded between the facesheets at the ends for gripping by hydraulic grips. Tabs are used at the ends to reduce the stress concentration caused by the grips during load application. Each face sheet was manufactured with four layers of IM7/977-2 4-harness satin fabric in a $[(0 / 90) / \pm 45 / \pm 45 /(0 / 90)]$ layup. The splice contained both IM7/977-2 tape and fabric. A potting compound (not shown) is used at the splice between the two sandwich sections. The specimen was impacted at the splice location with a $2.54 \mathrm{~cm}$ diameter indenter using $7.6 \mathrm{~J}$ of energy which displaced the surface $0.48 \mathrm{~mm}$ vertically. Flash thermography was performed on this specimen using the system described previously. Data was collected at a frame rate of 60 frames per second for 8 seconds after the flash heating. Conventional and model based fixed eigenvector PCA was performed on the data using a time window of from 0.08 to 1.5 seconds after heating. It should be noted that experimental-based fixed eigenvector PCA analysis was not performed because of insufficient experimental data sets for this specimen configuration. Figure 8 show the results of both data processing techniques as well as a pulse-echo ultrasonic attenuation image of the impact damage region. For reference the circles drawn on all three images are the same diameter (17.8mm) 
although the scaling of the thermal images is different from the ultrasonic image. The fixed eigenvector approach provided size information that more closely matched the ultrasonic results.

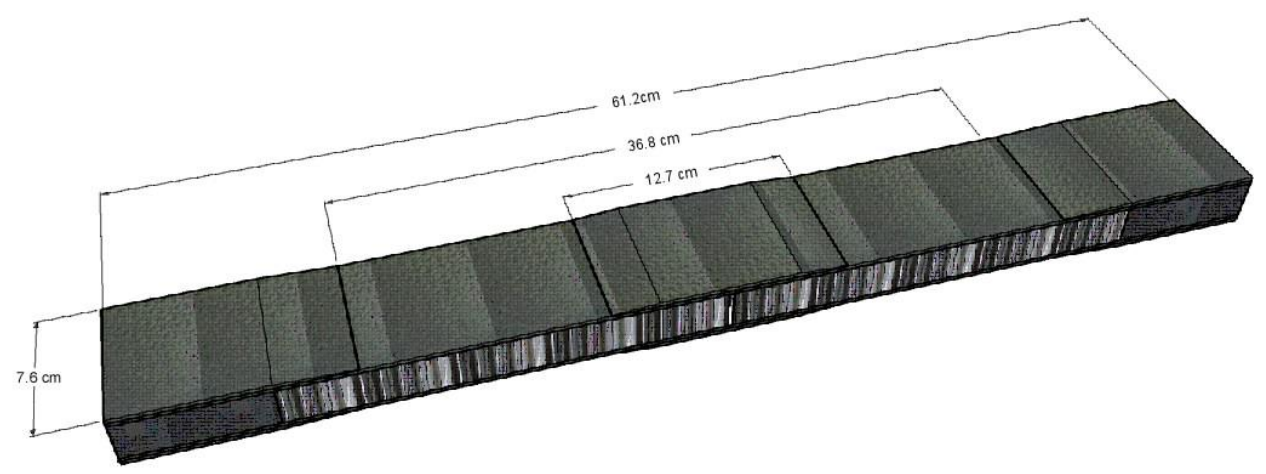

Figure 6. Drawing of the honeycomb sandwich specimen examined in this study.

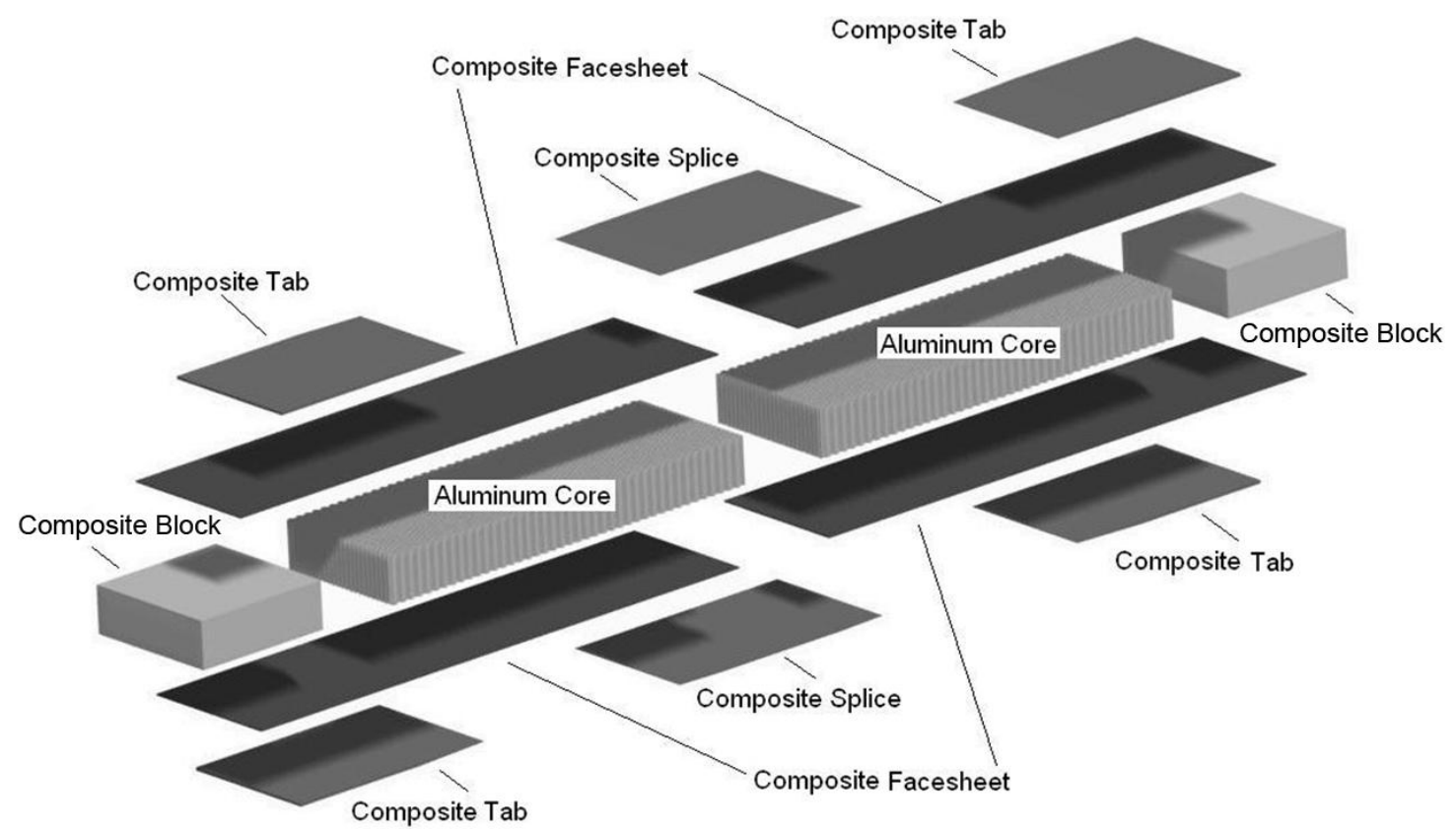

Figure 7. Exploded view of honeycomb sandwich specimen. 


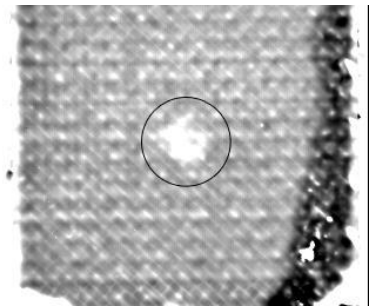

(a)

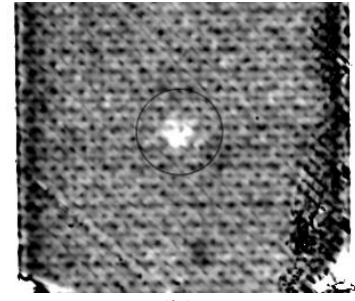

(b)

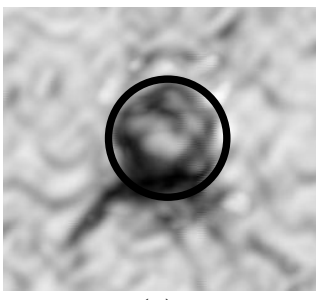

(c)

Figure 8. Two thermal and one ultrasonic image of the impact damage in a GrEp honeycomb specimen. (a) Model-based fixed eigenvector PCA image, (b) conventional PCA image and (c) pulse-echo ultrasonic attenuation image. The circles drawn for reference on all three images measure $17.8 \mathrm{~mm}$ in diameter.

\section{CONCLUSIONS}

A new approach to processing of thermal NDE data employing a fixed set of eigenvectors generated from a one dimensional analytic model of the thermal response of the material under investigation is presented. Application of this approach to data acquired from a flat-bottom hole specimen showed that the signal-to-noise ratio of the resulting images increased on average by $248 \%$ when processing with the model based fixed eigenvectors and $360 \%$ when processing with the experiment based eigenvectors as compared to conventional PCA. This improvement in signalto-noise may be attributed to the creation of the fixed eigenvector set from predominately defect free RCC. In the case of the model-based approach for RCC no defects were included. In the case of the experimental-based approach because a large cross-section of data was used to generate the eigenvectors, only a small percentage of that data contains the thermal response of defects. On the other hand, for the specimen considered here, conventional PCA included both undamaged and damaged regions of the material. The performance of the model based fixed eigenvector approach may be improved if the family of characteristic curves used to calculate the eigenvectors was increased to contain a small set of the thermal response of modeled defects. The model-based approach is useful in cases where only a limited number of specimens or experimental results are available.

Further, this technique was found to be useful for analysis of specimens whose geometry is sufficiently complex so as to require a model of higher dimensionality to accurately represent the thermal response. It has been shown that in cases where it is not practical, or possible, to create the higher dimensional model, fixed eigenvector analysis can still be performed using the results of a one dimensional model. For the GrEp honeycomb specimen examined in this study, the defect size measured after the model-based fixed eigenvector approach agreed more closely with the pulse-echo ultrasonic data, whereas the conventional PCA approach underestimated the defect size. It can also be noted that the increase signal-to-noise ratio observed in the RCC specimen case could be due to the more accurate sizing of the defects from the model-based fixed eigenvector approach.

\section{ACKNOWLEGEMENTS}

The authors would like to thank Jeffery Seebo of Lockheed-Martin Space Systems for his help in laboratory setup and data acquisition for the ultrasonic attenuation image.

\section{REFERENCES}

[1] K. Pearson, On lines and planes of closest fit to systems of points in space, The London, Edinburgh and Dublin Philosophical Magazine and Journal of Science 2: 559-572 (1901). 
[2] H. Hotelling, Analysis of a complex of statistical variable into principal components, Journal of Educational Psychology 24: 417-441 (1933).

[3] S. Vyas and L. Kumarankyake, Constructing socio-economic status indices: how to use principal components analysis, Health Policy Plan. 21 (6): 459-468 (2006).

[4] S.H. Heyer and F.P. Schloerb, Application of Principal Component Analysis to Large-scale Spectral Line Imaging Studies of the Interstellar Medium, The Astrophysical Journal 475: 173-187 (1997).

[5] R. Chellappa, C.L. Wilson, and S. Sirohey, Human and machine recognition of faces: A survey, Proceedings of the IEEE 83(5): 705-740 (1995).

[6] N. Rajic, Principal Component Thermography for Flaw Contrast Enhancement and Flaw Depth Characterisation in Composite Structures, Composite Structures 58: 521-528 (2002).

[7] N. Rajic Principal Component Thermography, DSTO-TR-1298 (2002).

[8] M.R. Valluzzi, et. al., IR thermography for interface analysis of FRP laminates externally bonded to RC beams, Materials and Structures 42:25-34 (2009).

[9] M. Genest, Image Processing for Automated Flaw Detection in Pulsed Thermography, Proceeding of 6th International Workshop-NDT Signal Processing: 1-9 (2009).

[10] V. P. Vavilov and S. Marinetti, Comparative Analysis of the Thermal Testing of Corrosion in High and Low Heat-Conducting Metals, Russian Journal of Nondestructive Testing 44(12): 841-846 (2008).

[11] J.N. Zalameda, P.A. Howell and W.P. Winfree, Compression Techniques for Improved Algorithm Computational Performance, Proceedings of SPIE, Thermosense XXVII, 5782: 399-406 (2005).

[12] S. Hermosilla-Lara, et. al., Enhancement of open-cracks detection using a principal component analysis/wavelet technique in photothermal nondestructive testing, Proceedings of Quantitative Infrared Thermography 6: 41-46 (2002).

[13] S. Marinetti et al., Statistical analysis of IR thermographic sequences by PCA, Infrared Physics \& Technology 46: 85-91 (2004).

[14] K.E. Cramer, et. al., Status of Thermal NDT of Space Shuttle Materials at NASA, Proc. SPIE 6205: 1B1-9 (2006).

[15] De Hoog, F.R., J.H. Knight, and A.N. Stokes, An improved method for numerical inversion of Laplace transforms. S.I.A.M. J. Sci. and Stat. Comput. 3: 357-366 (1982).

[16] Hollenbeck, K.J., INVLAP.M: A matlab function for numerical inversion of Laplace transforms by the De Hoog algorithm, available at http://www.isva.dtu.dk/staff/karl/invlap.htm, accessed Dec. 2002 (1998).

[17] K.E. Cramer, et. al., Review Of NDE Research And Development At NASA Langley Research Center In Support Of The Space Shuttle Return To Flight, Ensayos No Destructivos Y Estructurales, Neuquen: 1-6 (2005).

[18] K.E. Cramer and W.P. Winfree, The Application Of Infrared Thermographic Inspection Techniques To The Space Shuttle Thermal Protection System, Ensayos No Destructivos Y Estructurales, Neuquen: 227-233 (2005). 